\title{
New Branch of Intuitionistic Fuzzification in Algebras with Their Applications
}

\author{
Samaher Adnan Abdul-Ghani, Shuker Mahmood Khalil (D), \\ Mayadah Abd Ulrazaq, and Abu Firas Muhammad Jawad Al-Musawi
}

Department of Mathematics, College of Science, Basrah University, Basrah 61004, Iraq

Correspondence should be addressed to Shuker Mahmood Khalil; shuker.alsalem@gmail.com

Received 28 February 2018; Revised 22 May 2018; Accepted 11 June 2018; Published 10 July 2018

Academic Editor: Susana Montes

Copyright (c) 2018 Samaher Adnan Abdul-Ghani et al. This is an open access article distributed under the Creative Commons Attribution License, which permits unrestricted use, distribution, and reproduction in any medium, provided the original work is properly cited.

\begin{abstract}
The intuitionistic fuzzification in $\rho$-algebras about the concepts of ideals and subalgebras given with several related characterizations is considered. Some new concepts like intuitionistic fuzzy $\rho$-ideal (IF $\rho i$ ), intuitionistic fuzzy $\rho$-subalgebra (IF $\rho$ ), $\rho$-homomorphism, and intuitionistic fuzzy $\bar{\rho}$-ideal $(I F \bar{\rho} i)$ are introduced and some of their descriptions are given in this work. Further, we show some applications on the family of all intuitionistic fuzzy $\rho$-subalgebras $I F \rho_{S}(\Re)$ in $\rho$-algebra like the binary

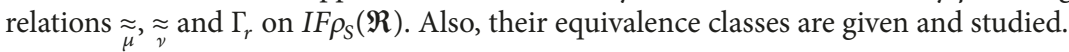

\section{Introduction}

The fuzzy set (FS) as suggested by Zadeh [1] in 1965 is a regulation to vagueness and encounter uncertainty. A FS maps each element of the universe of discourse to the interval $[0,1]$. After the introduction of fuzzy sets theory by him, many mathematicians were conducted on the generalizations of the this concept and studied in the groups, algebras, and soft spaces (see [2-5]). By including a fuzzy set the degree of nonmembership, Atanassov [6] in 1986 suggested the intuitionistic fuzzy set (IFS), which seems more precise for provides opportunities and uncertainty quantification to accurately model a problem based on existing knowledge and monitoring. Also, this notion is discussed in different fields (see [7-11])

$B C K$-algebra, class of algebra of logic, was investigated by Imai and Iseki [12]. After that, the notion of $d$-algebras was investigated by Neggers and Kim [13]. In 2017, the concepts of $\rho$-algebra, $\bar{\rho}$-ideal, $\rho$-ideal, $\rho$-subalgebra, and permutation topological $\rho$-algebra were first proposed by Mahmood and Abud Alradha [14]. Next, they showed the notion of the soft $\rho$-algebra and soft edge $\rho$-algebra [15].

In the present work, the notions of intuitionistic fuzzy $\rho$-ideal (IF $\rho$ i), intuitionistic fuzzy $\rho$-subalgebra (IF $\rho$ s), $\rho$-homomorphism, and intuitionistic fuzzy $\bar{\rho}$-ideal (IF $\bar{\rho} i)$ are introduced. Further, we show some applications on the family of all intuitionistic fuzzy $\rho$-subalgebras $I F \rho_{S}(\mathfrak{R})$ in $\rho$-algebra like the binary relations $\underset{\tilde{\mu}}{\widetilde{v}} \approx \widetilde{\tau}$ and $\Gamma_{r}$ on $I F \rho_{S}(\mathfrak{R})$. Also, their equivalence classes are given and studied.

\section{Preliminaries and Notations}

We will recall basic definitions and results to obtain properties developed in this work.

Definition 1 (see [16]). An intuitionistic fuzzy set $\alpha$ (IFS, in short) over the universe $\mathfrak{R}$ is defined by $\alpha=\{<$ $\left.a, \mu_{\alpha}(a), v_{\alpha}(a)>\mid a \in \mathfrak{R}\right\}$, where $\mu_{\alpha}(a): \Re \longrightarrow[0 ; 1]$, $v_{\alpha}(a): \Re \longrightarrow[0 ; 1]$ with $0 \leq \mu_{\alpha}(a)+v_{\alpha}(a) \leq 1, \forall a \in \Re$. $\mu_{\alpha}(a)$ and $v_{\alpha}(a)$ are real numbers and their values represent the degree of membership and nonmembership of $a$ to $\alpha$, respectively.

Definition 2 (see [6]). The IF whole and empty sets of $\Re$ are defined by $\overline{1}=\{\langle a,(1,0)\rangle \mid a \in \mathfrak{R}\}$ and $\overline{0}=\{\langle a,(0,1)\rangle \mid$ $a \in \mathfrak{R}\}$, respectively. 
2.1. Basic Relations and Operations on Intuitionistic Fuzzy Sets [7]. Assume $\alpha=\left\{\left\langle a,\left(\mu_{\alpha}(a), v_{\alpha}(a)\right)>\right| a \in \mathfrak{R}\right\}$ and $\beta=\{\prec$ $\left.a,\left(\mu_{\beta}(a), v_{\beta}(a)\right)>\mid a \in \mathfrak{R}\right\}$ are two IF sets of $\mathfrak{R}$. We deduced the following relations:

(1) [inclusion] $\alpha \subseteq \beta$ iff $\mu_{\alpha}(a) \leq \mu_{\beta}(a)$ and $v_{\alpha}(a) \geq v_{\beta}(a)$, $\forall a \in \mathfrak{R}$

(2) [equality] $\alpha=\beta$ iff $\alpha \subseteq \beta$ and $\beta \subseteq \alpha$,

(3) [intersection] $\alpha \widetilde{\cap} \beta=\left\{\left(a, \min \left\{\mu_{\alpha}(a), \mu_{\beta}(a)\right\}\right.\right.$, $\left.\left.\max \left\{v_{\alpha}(a), v_{\beta}(a)\right\}\right): a \in \mathfrak{R}\right\}$,

(4) [union] $\alpha \tilde{\cup} \beta=\left\{\left(a, \max \left\{\mu_{\alpha}(a), \mu_{\beta}(a)\right\}, \min \left\{v_{\alpha}(a)\right.\right.\right.$, $\left.\left.\left.v_{\beta}(a)\right\}\right): a \in \mathfrak{R}\right\}$

(5) [complement] $\alpha^{c}=\left\{\left(a, v_{\alpha}(a), \mu_{\alpha}(a)\right), a \in \mathfrak{R}\right\}$.

Definition 3 (see [14]). We say $(\mathfrak{R}, \bullet, 0)$ is $\rho$-algebra if $(\bullet)$ is a binary operation on $\mathfrak{R}$ with a constant $0 \in \mathfrak{R}$ and such that

(1) $a \cdot a=0$,

(2) $0 \cdot a=0$,

(3) $a \cdot b=0=b \cdot a$ imply that $a=b$,

(4) For all $a \neq b \in \mathfrak{R}-\{0\}$ imply that $a \bullet b=b \bullet a \neq 0$.

Definition 4 (see [14]). Assume $(\Re, \bullet, 0)$ is a $\rho$-algebra and $\phi \neq \mathrm{K} \subseteq \mathfrak{R}$. We say $K$ is a $\rho$-subalgebra of $\mathfrak{R}$ if $a \bullet b \in K$, $\forall a, b \in K$.

Definition 5 (see $[14])$. Assume $(\mathfrak{R}, \bullet, 0)$ is $\rho$-algebra and $\phi \neq$ $K \subseteq \mathfrak{R}$. We say $K$ is $\rho$-ideal of $\mathfrak{R}$ if

(1) $a, b \in K$ imply $a \bullet b \in K$,

(2) $a \cdot b \in K$ and $b \in K$ imply $a \in K, \forall a, b \in \Re$.

Definition 6 (see [14]). Assume $(\Re, \bullet, 0)$ is a $\rho$-algebra and $K$ subset of $\mathfrak{R}$. We say $K$ is a $\bar{\rho}$-ideal of $\mathfrak{R}$ if

(1) $0 \in K$,

(2) $a \in K$ and $b \in \mathfrak{R} \longrightarrow a \bullet b \in K, \forall a, b \in \mathfrak{R}$.

Definition 7 (see [11]). Assume that $\alpha=\left\{<a,\left(\mu_{\alpha}(a)\right.\right.$, $\left.\left.v_{\alpha}(a)\right)>\mid a \in \mathfrak{R}\right\}$ is an IFS in $\mathfrak{R}$ and $r \in[0,1]$. The set $W\left(\mu_{\alpha}, r\right)=\left\{a \in \mathfrak{R} \mid \mu_{\alpha}(a) \geq r\right\}$ (resp., $L\left(\nu_{\alpha}, r\right)=\{a \in \mathfrak{R} \mid$ $\left.\nu_{\alpha}(a) \leq r\right\}$ ) is said to be $\mu$-level $r$-cut (resp., $\nu$-level $r$-cut) of $\alpha$.

\section{Intuitionistic Fuzzy $\rho$-Subalgebras in $\rho$-Algebras}

In this section, we introduce some new concepts, such as (IF $\rho$ ), (IF $\rho$ i), (IF $\rho i)$, and $\rho$-homomorphism which are introduced and discussed. Further, some binary relations $\underset{\mu}{\widetilde{\mu}}$, $\approx \underset{\nu}{ }$ and $\Gamma_{r}$ on $I F \rho_{S}(\mathfrak{R})$ are given, and some basic properties are shown.

Definition 8. Assume $(\Re, \bullet, 0)$ is a $\rho$-algebra and $\alpha=\{\prec$ $\left.a,\left(\mu_{\alpha}(a), v_{\alpha}(a)\right)>\mid a \in \Re\right\}$ is IFS of $\mathfrak{R}$. We say $\alpha$ is an (IF $\rho)$ of $\Re$ if $\mu_{\alpha}(a \cdot b) \geq \min \left\{\mu_{\alpha}(a), \mu_{\alpha}(b)\right\}$ and $\nu_{\alpha}(a \bullet b) \leq$ $\max \left\{v_{\alpha}(a), v_{\alpha}(b)\right\}, \forall a, b \in \mathfrak{R}$.

\begin{tabular}{lcccc}
\multicolumn{5}{c}{ TABLE 1} \\
\hline$*$ & 0 & $\omega$ & $\partial$ & $\ell$ \\
0 & 0 & 0 & 0 & 0 \\
$\omega$ & $\omega$ & 0 & $\partial$ & $\partial$ \\
$\partial$ & $\omega$ & $\partial$ & 0 & $\omega$ \\
$\ell$ & $\ell$ & $\partial$ & $\omega$ & 0 \\
\hline
\end{tabular}

Example 9. Let $\mathfrak{R}=\{0, \omega, \partial, \ell\}$ be $\rho$-algebra with Table 1 .

Then, $\alpha=\left\{\left\langle a,\left(\mu_{\alpha}(a), \nu_{\alpha}(a)\right)>\right| a \in \mathfrak{R}\right\}=\{(0,0.9$, $0.1),(\omega, 0.4,0.3),(\partial, 0.7,0.3),(\ell, 0.4,0.2)\}$ is an $(I F \rho s)$ of $\Re$.

Definition 10. Assume $(\mathfrak{R}, \bullet, 0)$ is $\rho$-algebra and $\alpha=\{<$ $\left.a,\left(\mu_{\alpha}(a), \nu_{\alpha}(a)\right)>\mid a \in \mathfrak{R}\right\}$ is IFS of $\mathfrak{R}$. We say $\alpha$ is (IF $\rho$ i) of $\Re$ if

(1) $\mu_{\alpha}(a \cdot b) \geq \min \left\{\mu_{\alpha}(a), \mu_{\alpha}(b)\right\}$ and $\nu_{\alpha}(a \cdot b) \leq$ $\max \left\{v_{\alpha}(a), v_{\alpha}(b)\right\}$

(2) $\mu_{\alpha}(a) \geq \min \left\{\mu_{\alpha}(a \bullet b), \mu_{\alpha}(b)\right\}$ and $\nu_{\alpha}(a) \leq \max \left\{\nu_{\alpha}(a \bullet\right.$ b), $\left.\nu_{\alpha}(b)\right\}, \forall a, b \in \mathfrak{R}$.

Example 11. Let $(\Re, \bullet, 0)$ be $\rho$-algebra in Example 9 and let $\alpha=\left\{\left\langle a,\left(\mu_{\alpha}(a), \nu_{\alpha}(a)\right)>\right| a \in \mathfrak{R}\right\}=\{(0,0.8,0.2)$, $(\omega, 0.3,0.4),(\partial, 0.2,0.7),(\ell, 0.4,0.3)\}$ be IFS of $\mathfrak{R}$. Then, $\alpha$ is (IF $\rho$ i) of $\mathfrak{R}$.

Definition 12. Assume $(\mathfrak{R}, \bullet, 0)$ is $\rho$-algebra and $\alpha=\{\prec a$, $\left.\left(\mu_{\alpha}(a), \nu_{\alpha}(a)\right)>\mid a \in \mathfrak{R}\right\}$ is IFS of $\mathfrak{R}$. We say $\alpha$ is $(I F \bar{\rho} i)$ of $\mathfrak{R}$ if

(1) $\mu_{\alpha}(0) \geq \mu_{\alpha}\left(\right.$ a) and $\nu_{\alpha}(0) \leq \nu_{\alpha}(a)$,

(2) $\mu_{\alpha}(a \cdot b) \geq \min \left\{\mu_{\alpha}(a), \mu_{\alpha}(b)\right\}$ and $\nu_{\alpha}(a \cdot b) \leq$ $\max \left\{v_{\alpha}(a), v_{\alpha}(b)\right\}, \forall a, b \in \mathfrak{R}$.

Example 13. Let $(\Re, \bullet, 0)$ be $\rho$-algebra in Example 9 and let $\alpha=\left\{\prec a,\left(\mu_{\alpha}(a), \nu_{\alpha}(a)\right)>\mid a \in \mathfrak{R}\right\}=\{(0,0.9,0.1)$, $(\omega, 0.4,0.3),(\partial, 0.7,0.3),(\ell, 0.4,0.2)\}$ be IFS of $\mathfrak{R}$. Then, $\alpha$ is (IF $\bar{\rho} i)$ of $\Re$.

Remark 14.

(1) If $\alpha=\left\{\prec a,\left(\mu_{\alpha}(a), \nu_{\alpha}(a)\right)>\mid a \in \mathfrak{R}\right\}$ is (IF $\rho$ i) of $\mathfrak{R}$, then $\alpha$ is $(I F \rho s)$.

(2) If $\alpha=\left\{\prec a,\left(\mu_{\alpha}(a), v_{\alpha}(a)\right)>\mid a \in \Re\right\}$ is $(I F \bar{\rho} i)$ of $\mathfrak{R}$, then $\alpha$ is $(I F \rho s)$.

(3) If $\alpha=\left\{\prec a,\left(\mu_{\alpha}(a), \nu_{\alpha}(a)\right)>\mid a \in \mathfrak{R}\right\}$ is (IF $\rho$ s) of $\mathfrak{R}$ and satisfies (2) in Definition 10, then $\alpha$ is (IF $\rho$ i).

(4) If $\alpha=\left\{\prec a,\left(\mu_{\alpha}(a), \nu_{\alpha}(a)\right)>\mid a \in \mathfrak{R}\right\}$ is (IF $\rho$ s) of $\mathfrak{R}$ and satisfies (1) in Definition 12, then $\alpha$ is $(I F \bar{\rho} i)$.

Lemma 15. If $\alpha=\left\{\left\langle a,\left(\mu_{\alpha}(a), v_{\alpha}(a)\right)>\right| a \in \mathfrak{R}\right\}$ is (IF $\rho$ s) of $\mathfrak{R}$, then $\mu_{\alpha}(0) \geq \mu_{\alpha}(a)$ and $\nu_{\alpha}(0) \leq \nu_{\alpha}(a), \forall a \in \mathfrak{R}$.

Proof. Let $a \in \mathfrak{R}$. Then $\mu_{\alpha}(0)=\mu_{\alpha}(a \cdot a) \geq$ $\min \left\{\mu_{\alpha}(a), \mu_{\alpha}(a)\right\}=\mu_{\alpha}(a)$ and $\nu_{\alpha}(0)=v_{\alpha}(a \cdot a) \leq$ $\max \left\{v_{\alpha}(a), v_{a}(a)\right\}=v_{\alpha}(a)$. 
Theorem 16. If $\left\{\alpha_{i}=\prec a,\left(\mu_{\alpha_{i}}(a), \nu_{\alpha_{i}}(a)\right)>\mid a \in \Re, i \in I\right\}$ is

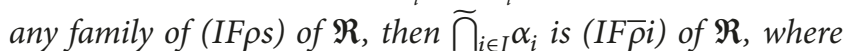
$\widetilde{\bigcap}_{i \in I} \alpha_{i}=\left\{\prec a,\left(\min \left\{\mu_{\alpha_{i}}(a)\right\}, \max \left\{\nu_{\alpha_{i}}(a)\right\}\right)>\mid a \in \mathfrak{R}\right\}$.

Proof. Let $a, b \in \Re$. Thus we consider that $\min \left\{\mu_{\alpha_{i}}(a \cdot b)\right\} \geq \min \left\{\min \left\{\mu_{\alpha_{i}}(a), \mu_{\alpha_{i}}(b)\right\}\right\}=$ $\min \left\{\min \left\{\mu_{\alpha_{i}}(a)\right\}, \min \left\{\mu_{\alpha_{i}}(b)\right\}\right\}$. Also $\max \left\{\nu_{\alpha_{i}}(a \bullet b)\right\} \leq$ $\max \left\{\max \left\{\nu_{\alpha_{i}}(a), \nu_{\alpha_{i}}(b)\right\}\right\}=\max \left\{\max \left\{\nu_{\alpha_{i}}(a)\right\}, \max \left\{\nu_{\alpha_{i}}(b)\right\}\right\}$.

Thus $\widetilde{\bigcap}_{i \in I} \alpha_{i}=\left\{\prec a,\left(\min \left\{\mu_{\alpha_{i}}(a)\right\}, \max \left\{\nu_{\alpha_{i}}(a)\right\}\right)>\mid a \in\right.$ $\mathfrak{R}\}$ satisfies condition (2) in Definition 12. Also, let $a \in$ $\mathfrak{R}$. Hence, we consider that $\min \left\{\mu_{\alpha_{i}}(0)\right\}=\min \left\{\mu_{\alpha_{i}}(a\right.$ • a) $\geq \geq \min \left\{\mu_{\alpha_{i}}(a), \mu_{\alpha_{i}}(a)\right\}=\min \left\{\mu_{\alpha_{i}}(a)\right\}$. Furthermore, $\max \left\{v_{\alpha_{i}}(0)\right\}=\max \left\{\nu_{\alpha_{i}}(a \cdot a)\right\} \leq \max \left\{\nu_{\alpha_{i}}(a), \nu_{\alpha_{i}}(a)\right\}=$ $\max \left\{\nu_{\alpha_{i}}(a)\right\}$. Then (1) in Definition 12 is held and hence $\widetilde{\bigcap}_{i \in I} \alpha_{i}$ is $(I \bar{\rho} i)$ of $\mathfrak{R}$.

Theorem 17. If $\alpha=\left\{\left\langle a,\left(\mu_{\alpha}(a), v_{\alpha}(a)\right) \succ\right| a \in \mathfrak{R}\right\}$ is (IF $\left.\rho\right)$ of $\mathfrak{R}$, then $K=\prec a, \mu_{\alpha}(a), 1-\mu_{\alpha}(a)>$ is (IF $\rho$ i) of $\mathfrak{R}$.

Proof. We need only to show that $1-\mu_{\alpha}(a)$ satisfies the first and second condition in Definition 10. Assume $\forall a, b \in \mathfrak{R}$. Then $1-\mu_{\alpha}(a \cdot b) \leq 1-\min \left\{\mu_{\alpha}(a), \mu_{\alpha}(b)\right\}=\max \{1-$ $\left.\mu_{\alpha}(a), 1-\mu_{\alpha}(b)\right\}$. Furthermore, $1-\mu_{\alpha}(a) \leq 1-\min \left\{\mu_{\alpha}(a\right.$ • $\left.b), \mu_{\alpha}(b)\right\}=\max \left\{1-\mu_{\alpha}(a \bullet b), 1-\mu_{\alpha}(b)\right\}$. Hence $K$ is (IF $\left.\rho\right)$ of $\mathfrak{R}$.

Theorem 18. If $\alpha=\left\{\left\langle a,\left(\mu_{\alpha}(a), \nu_{\alpha}(a)\right)\right\rangle \mid a \in \mathfrak{R}\right\}$ is (IF $\rho$ s) of $\mathfrak{R}$, then the sets $T_{\mu}=\left\{a \in \mathfrak{R} \mid \mu_{\alpha}(a)=\mu_{\alpha}(0)\right\}$ and $T_{v}=\{a \in$ $\left.\mathfrak{R} \mid \nu_{\alpha}(a)=\nu_{\alpha}(0)\right\}$ are $\rho$-subalgebras of $\mathfrak{R}$.

Proof. Let $a, b \in T_{\mu}$. Hence $\mu_{\alpha}(a)=\mu_{\alpha}(0)=\mu_{\alpha}(b)$, and $\mu_{\alpha}(a \bullet b) \geq \min \left\{\mu_{\alpha}(a), \mu_{\alpha}(b)\right\}=\mu_{\alpha}(0)$. By using Lemma 15, we consider that $\mu_{\alpha}(a \bullet b)=\mu_{\alpha}(0)$ or equivalently $a \bullet b \in T_{\mu}$. Now, let $a, b \in T_{v}$. This implies that $\nu_{\alpha}(a \bullet b) \leq \max \left\{\nu_{\alpha}(a), \nu_{\alpha}(b)\right\}=$ $\nu_{\alpha}(0)$ and, by applying Lemma 15 , we conclude that $\nu_{\alpha}(a \bullet b)=$ $\nu_{\alpha}(0)$. Therefore $a \bullet b \in T_{\nu}$.

Definition 19. Assume $\alpha=\left\{\left\langle a,\left(\mu_{\alpha}(a), \nu_{\alpha}(a)\right)\right\rangle \mid a \in \mathfrak{R}\right\}$ is (IF $\rho$ S) of $\mathfrak{R}$. We say $\alpha$ has finite image, if each image of $\mu_{\alpha}$ and $\nu_{\alpha}$ is with finite cardinality (i.e., $\operatorname{Im}\left(\mu_{\alpha}\right)=\left\{\mu_{\alpha}(a) \mid a \in \mathfrak{R}\right\}$ and $\operatorname{Im}\left(\nu_{\alpha}\right)=\left\{\nu_{\alpha}(a) \mid a \in \mathfrak{R}\right\}$ such that $\left|\operatorname{Im}\left(\mu_{\alpha}\right)\right|<\infty$ and $\left.\left|\operatorname{Im}\left(\nu_{\alpha}\right)\right|<\infty\right)$.

Definition 20. Assume that $\alpha=\left\{\left\langle a,\left(\mu_{\alpha}(a), v_{\alpha}(a)\right)\right\rangle \mid a \in\right.$ $\mathfrak{R}\}$ is (IF $\rho)$ of $\mathfrak{R}$ and $r \in[0,1]$. The set $W\left(\mu_{\alpha}, r\right)=\{a \in \mathfrak{R} \mid$ $\left.\mu_{\alpha}(a) \geq r\right\}$ (resp., $L\left(\nu_{\alpha}, r\right)=\left\{a \in \Re \mid \nu_{\alpha}(a) \leq r\right\}$ ) is said to be $\mu$-level $r$-cut (resp., $\nu$-level $r$-cut) of $\alpha$.

Theorem 21. If $\alpha=\left\{\left\langle a,\left(\mu_{\alpha}(a), \nu_{\alpha}(a)\right)>\right| a \in \mathfrak{R}\right\}$ is (IF $\rho$ s) of $\mathfrak{R}$, then $W\left(\mu_{\alpha}, r\right)=\left\{a \in \mathfrak{R} \mid \mu_{\alpha}(a) \geq r\right\}$ and $L\left(\nu_{\alpha}, r\right)=\{a \in$ $\left.\left.\mathfrak{R} \mid \nu_{\alpha}(a) \leq r\right\}\right)$ of $\alpha$ are $\rho$-subalgebras of $\mathfrak{R}$.

Proof. Let $a, b \in W\left(\mu_{\alpha}, r\right)$. Hence $\mu_{\alpha}(b) \geq r$ and $\mu_{\alpha}(b) \geq r$. This implies that $\mu_{\alpha}(a \bullet b) \geq \min \left\{\mu_{\alpha}(a), \mu_{\alpha}(b)\right\} \geq r$ so that $a \cdot b \in W\left(\mu_{\alpha}, r\right)$. Thus $W\left(\mu_{\alpha}, r\right)$ is $\rho$-subalgebra of $\mathfrak{R}$ Now let $a, b \in L\left(\nu_{\alpha}, r\right)$. Thus $\nu_{\alpha}(a \bullet b) \leq \max \left\{\nu_{\alpha}(a), \nu_{\alpha}(b)\right\} \leq r$ and $a \cdot b \in L\left(\nu_{\alpha}, r\right)$. Therefore $L\left(\nu_{\alpha}, r\right)$ is $\rho$-subalgebra of $\mathfrak{R}$.
Theorem 22. If $\alpha=\left\{\left\langle a,\left(\mu_{\alpha}(a), \nu_{\alpha}(a)\right)>\right| a \in \mathfrak{R}\right\}$ is IFS of $\rho$ - algebra $\mathfrak{R}$ such that the sets $W\left(\mu_{\alpha}, r\right)$ and $L\left(\nu_{\alpha}, r\right)$ are $\rho$-subalgebras of $\Re$, then $\alpha$ is an (IF $\rho$ s) of $\mathfrak{R}$.

Proof. Suppose that there are two members $t_{1}$ and $t_{2}$ in $\mathfrak{R}$ with $\mu_{\alpha}\left(t_{1} \cdot t_{2}\right)<\min \left\{\mu_{\alpha}\left(t_{1}\right), \mu_{\alpha}\left(t_{2}\right)\right\}$. Let $t=\left[\mu_{\alpha}\left(t_{1} \bullet\right.\right.$ $\left.\left.t_{2}\right)+\min \left\{\mu_{\alpha}\left(t_{1}\right), \mu_{\alpha}\left(t_{2}\right)\right\}\right] / 2$. Hence $\mu_{\alpha}\left(t_{1} \cdot t_{2}\right)<t<$ $\min \left\{\mu_{\alpha}\left(t_{1}\right), \mu_{\alpha}\left(t_{2}\right)\right\}$ and so $t_{1} \bullet t_{2} \notin W\left(\mu_{\alpha}, t\right)$, but $t_{1}, t_{2} \in$ $W\left(\mu_{\alpha}, t\right)$. This is a contradiction, and therefore $\mu_{\alpha}(a \bullet b) \geq$ $\min \left\{\mu_{\alpha}(a), \mu_{\alpha}(b)\right\}, \forall a, b \in \mathfrak{R}$. Now assume that $\nu_{\alpha}\left(t_{1} \bullet t_{2}\right)>$ $\min \left\{v_{\alpha}\left(t_{1}\right), v_{\alpha}\left(t_{2}\right)\right\}$ for some $t_{1}, t_{2} \in \mathfrak{R}$. Taking $k=\left[v_{\alpha}\left(t_{1} \bullet\right.\right.$ $\left.\left.t_{2}\right)+\min \left\{v_{\alpha}\left(t_{1}\right), v_{\alpha}\left(t_{2}\right)\right\}\right] / 2$, then we consider that $\nu_{\alpha}\left(t_{1} \bullet t_{2}\right)>$ $k>\max \left\{v_{\alpha}\left(t_{1}\right), v_{\alpha}\left(t_{2}\right)\right\}$. It follows that $t_{1}, t_{2} \in L\left(v_{\alpha}, k\right)$ and $t_{1} \cdot t_{2} \notin L\left(v_{\alpha}, k\right)$. This is a contradiction. Therefore, we consider that $\nu_{\alpha}(a \bullet b) \leq \max \left\{\nu_{\alpha}(a), \nu_{\alpha}(b)\right\}, \forall a, b \in \mathfrak{R}$. Then $A$ is (IF $\rho$ s) of $\mathfrak{R}$.

Theorem 23. If $H$ is $\rho$-subalgebra of $\mathfrak{R}$, then there exists (IF $\rho$ s) $\alpha$ of $\mathfrak{R}$, where $H$ satisfies both $\mu$-level $\rho$-subalgebra and $\nu$-level $\rho$-subalgebra of $\alpha$ in $\mathfrak{R}$.

Proof. Assume $H$ is $\rho$-subalgebra of $\Re$ and let $\mu_{\alpha}$ and $\nu_{\alpha}$ be fuzzy sets in $\mathfrak{R}$ defined by

$$
\mu_{\alpha}(a)= \begin{cases}k, & \text { if } a \in H \\ 1, & \text { Otherwise }\end{cases}
$$

and

$$
v_{\alpha}(a)= \begin{cases}m, & \text { if } \alpha \in H \\ 1, & \text { Otherwise }\end{cases}
$$

$\forall a \in \Re$, where $k, m \in(0,1)$ are fixed real numbers with $k+m<1$. Assume $a, b \in \Re$. Then $a \cdot b \in H$ whenever $a, b \in H$. This implies that $\mu_{\alpha}(a \bullet b)=\min \left\{\mu_{\alpha}(a), \mu_{\alpha}(b)\right\}$ and $\nu_{\alpha}(a \bullet b) \leq \max \left\{v_{\alpha}(a), v_{\alpha}(b)\right\}$. If at least one of $a$ or $b$ does not belong to $H$, then either $\mu_{\alpha}(a)=0$ or $\mu_{\alpha}(b)=0$ and hence either $\nu_{\alpha}(a)=1$ or $\nu_{\alpha}(b)=1$. It follows that $\mu_{\alpha}(a \bullet b) \geq 0=$ $\min \left\{\mu_{\alpha}(a), \mu_{\alpha}(b)\right\}, \nu_{\alpha}(a \bullet b) \leq 1=\max \left\{\nu_{\alpha}(a), v_{\alpha}(b)\right\}$. Hence $\alpha=\left\{\left\langle a,\left(\mu_{\alpha}(a), \nu_{\alpha}(a)\right)>\right| a \in \mathfrak{R}\right\}$ is (IF $\left.\rho\right)$ of $\mathfrak{R}$. Obviously, $W\left(\mu_{\alpha}, k\right)=H=L\left(\nu_{\alpha}, m\right)$.

Definition 24. Assume $\Theta: \Re \longrightarrow Y$ is a mapping of $\rho$-algebras. We say $\Theta$ is $\rho$-homomorphism if $\Theta(a \bullet$ b) $=\Theta(a) \cdot \Theta(b), \forall a, b \in \Re$. And $\Theta^{-1}(\beta)=\{<$ $\left.a,\left(\Theta^{-1} \mu_{\beta}(a), \Theta^{-1} v_{\beta}(a)\right)>\mid a \in \Re\right\}$ is IFS in $\rho$-algebra $\mathfrak{R}$ for any IFS $\beta=\left\{\left\langle c,\left(\mu_{\beta}(c), v_{\beta}(c)\right)\right\rangle \mid c \in Y\right\}$ of $\rho$-algebra $Y$. Also, if $\alpha=\left\{\left\langle a,\left(\mu_{\alpha}(a), v_{\alpha}(a)\right)>\right| a \in \mathfrak{R}\right\}$ is IFS in $\rho$-algebra $\mathfrak{R}$, then $\Theta(\alpha)$ is IFS in $Y$ and defined by

$\Theta(\alpha)=\left\{\prec c,\left(\Theta_{\text {sup }} \mu_{\alpha}(y), \Theta_{\text {inf }} \nu_{\alpha}(c)\right)>\mid c \in Y\right\}$, where

$$
\begin{aligned}
& \Theta_{\text {sup }} \mu_{\alpha}(c) \\
& \quad= \begin{cases}\sup \left\{\mu_{\alpha}(a) \mid a \in \Theta^{-1}(c)\right\}, & \text { if } \Theta^{-1}(c) \neq 0, \\
0, & \text { Otherwise, }\end{cases}
\end{aligned}
$$


and

$$
\begin{aligned}
& \Theta_{\mathrm{inf}} v_{\alpha}(c) \\
& =\left\{\begin{array}{ll}
\inf \left\{v_{\alpha}(a) \mid a \in \Theta^{-1}(c)\right\}, & \text { if } \Theta^{-1}(c) \neq 0, \\
1, & \text { Otherwise, }
\end{array},\right.
\end{aligned}
$$

$\forall c \in Y$.

Theorem 25. Let $\Theta$ be $\rho$-homomorphism of $\rho$-algebra $\mathfrak{R}$ into $\rho$-algebra $Y$ and $K$ be (IF $\rho$ s) of $Y$. Then $\Theta^{-1}(K)$ is (IF $\rho$ s) of $\mathfrak{R}$.

Proof. Assuming $a, b \in \Re$, we have $\mu_{\Theta^{-1}(K)}(a \bullet b)=\mu_{K}(\Theta(a \bullet$ b) $)=\mu_{K}(\Theta(a) \cdot \Theta(b)) \geq \min \left\{\mu_{K}(\Theta(a)), \mu_{K}(\Theta(b))\right\}=$ $\min \left\{\mu_{\Theta^{-1}(K)}(a), \mu_{\Theta^{-1}(K)}(b)\right\}$ and $\nu_{\Theta^{-1}(K)}(a \bullet b)=v_{K}(\Theta(a \bullet$ $b))=v_{K}(\Theta(a) \cdot \Theta(b)) \leq \max \left\{v_{K}(\Theta(a)), v_{K}(\Theta(b))\right\}=$ $\max \left\{\nu_{\Theta^{-1}(K)}(a), v_{\Theta^{-1}(K)}(b)\right\}$. Thus $\Theta^{-1}(K)$ is $(I F \rho s)$ of $\mathfrak{R}$.

Theorem 26. Assume $\Theta: \mathfrak{R} \longrightarrow Y$ is $\rho$-homomorphism of $\rho$-algebra $\mathfrak{R}$ into $\rho$-algebra $Y$ and $\alpha=\left\{\left\langle a,\left(\mu_{\alpha}(a), \nu_{\alpha}(a)\right)\right\rangle \mid\right.$ $a \in \mathfrak{R}\}$ is (IF $\rho$ s) of $\mathfrak{R}$. Then $\Theta(\alpha)=\prec b,\left(\Theta_{\text {sup }}\left(\mu_{\alpha}\right), \Theta_{\text {inf }}\left(\nu_{\alpha}\right)\right)>$ is (IF $\rho$ ) of $Y$.

Proof. Let $\alpha=\left\{\left\langle a,\left(\mu_{\alpha}(a), \nu_{\alpha}(a)\right)>\right| a \in \mathfrak{R}\right\}$ be (IF $\rho s$ ) of $\mathfrak{R}$ and let $t_{1}, t_{2} \in Y$. Noticing that $\left\{a_{1} \bullet a_{2} \mid\right.$ $a_{1} \in \Theta^{-1}\left(t_{1}\right)$ and $\left.a_{2} \in \Theta^{-1}\left(t_{2}\right)\right\} \subseteq\{a \in \Re \mid a \epsilon$ $\left.\Theta^{-1}\left(t_{1} \cdot t_{2}\right)\right\}$, we have $\Theta_{\text {sup }}\left(\mu_{\alpha}\right)\left(t_{1} \cdot t_{2}\right)=\sup \left\{\mu_{\alpha}(a) \mid a \in\right.$ $\left.\Theta^{-1}\left(t_{1} * t_{2}\right)\right\} \geq \sup \left\{\mu_{\alpha}\left(a_{1} \cdot a_{2}\right) \mid a_{1} \in \Theta^{-1}\left(t_{1}\right)\right.$ and $a_{2} \in$ $\left.\Theta^{-1}\left(t_{2}\right)\right\} \geq \sup \left\{\min \left\{\mu_{\alpha}\left(a_{1}\right), \mu_{\alpha}\left(a_{2}\right)\right\} \mid a_{1} \in \Theta^{-1}\left(t_{1}\right)\right.$ and $a_{2} \in$ $\left.\Theta^{-1}\left(t_{2}\right)\right\}=\min \left\{\sup \left\{\mu_{\alpha}\left(a_{1}\right) \quad \mid a_{1} \in \Theta^{-1}\left(t_{1}\right)\right\}, \sup \left\{\mu_{\alpha}\left(a_{2}\right) \mid\right.\right.$ $\left.\left.a_{2} \in \Theta^{-1}\left(t_{2}\right)\right\}\right\}=\min \left\{\Theta_{\text {sup }}\left(\mu_{\alpha}\right)\left(t_{1}\right), \Theta_{\text {sup }}\left(\mu_{\alpha}\right)\left(t_{2}\right)\right\}$. Also, we consider that $\Theta_{\text {inf }}\left(\nu_{\alpha}\right)\left(t_{1} \bullet t_{2}\right)=\inf \left\{v_{\alpha}(a) \mid a \in \Theta^{-1}\left(t_{1} \bullet\right.\right.$ $\left.\left.t_{2}\right)\right\} \leq \inf \left\{v_{\alpha}\left(a_{1} \cdot a_{2}\right) \mid a_{1} \in \Theta^{-1}\left(t_{1}\right)\right.$ and $\left.a_{2} \in \Theta^{-1}\left(t_{2}\right)\right\} \leq$ $\inf \left\{\max \left\{v_{\alpha}\left(a_{1}\right), v_{\alpha}\left(a_{2}\right)\right\} \mid a_{1} \in \Theta^{-1}\left(t_{1}\right)\right.$ and $\left.a_{2} \in \Theta^{-1}\left(t_{2}\right)\right\}$ $=\max \left\{\inf \left\{v_{\alpha}\left(a_{1}\right) \mid a_{1} \in \Theta^{-1}\left(t_{1}\right)\right\}, \inf \left\{v_{\alpha}\left(a_{2}\right) \quad \mid a_{2} \epsilon\right.\right.$ $\left.\left.\Theta^{-1}\left(t_{2}\right)\right\}\right\}=\max \left\{\Theta_{\text {sup }}\left(\nu_{\alpha}\right)\left(t_{1}\right), \Theta_{\text {sup }}\left(\nu_{\alpha}\right)\left(t_{2}\right)\right\}$. Hence $\Theta(\alpha)=\prec$ $b,\left(\Theta_{\text {sup }}\left(\mu_{\alpha}\right), \Theta_{\text {inf }}\left(\nu_{\alpha}\right)\right)>$ is $($ IF $\rho s)$ of $Y$.

Theorem 27. Assume $\Theta: \mathfrak{R} \longrightarrow Y$ is $\rho$-homomorphism of $\rho$-algebra $\mathfrak{R}$ into $\rho$-algebra $Y$ and $\alpha=\left\{\left\langle a,\left(\mu_{\alpha}(a), \nu_{\alpha}(a)\right)>1\right.\right.$ $a \in \mathfrak{R}\}$ is (IF $\rho$ i) of $\mathfrak{R}$. Then $\Theta(\alpha)=<b,\left(\Theta_{\text {sup }}\left(\mu_{\alpha}\right), \Theta_{\text {inf }}\left(\nu_{\alpha}\right)\right)>$ is (IF $\rho$ i) of $Y$.

Proof. Since $\alpha=\left\{\left\langle a,\left(\mu_{\alpha}(a), \nu_{\alpha}(a)\right)>\right| a \in \mathfrak{R}\right\}$ is (IF $\left.\rho i\right)$ of $\mathfrak{R}$, then by Theorem 26 and Remark 14 we have $\Theta(\alpha)=\prec$ $b,\left(\Theta_{\text {sup }}\left(\mu_{\alpha}\right), \Theta_{\text {inf }}\left(\nu_{\alpha}\right)\right)>$ as $($ IF $\rho i)$ of $Y$. Hence condition (1) in Definition 10 is held. Since $\Theta$ is surjective, then for any $t_{1}, t_{2} \in Y, \exists a_{1}, a_{2} \in \mathfrak{R}$ such that $a_{1} \in \Theta^{-1} \Theta\left(a_{1}\right)=\Theta^{-1}\left(t_{1}\right)$ and $a_{2} \in \Theta^{-1} \Theta\left(a_{2}\right)=\Theta^{-1}\left(t_{2}\right)$. Also, $a_{1} \cdot a_{2} \in \Theta^{-1}\left(t_{1}\right) \cdot$ $\Theta^{-1}\left(t_{1}\right)=\Theta^{-1}\left(t_{1} \cdot t_{1}\right)$. Further, noticing that $\mu_{\alpha}\left(a_{1}\right) \geq$ $\min \left\{\mu_{\alpha}\left(a_{1} \bullet a_{2}\right), \mu_{\alpha}\left(a_{2}\right)\right\}$ and $\nu_{\alpha}\left(a_{1}\right) \leq \max \left\{v_{\alpha}\left(a_{1} \bullet a_{2}\right), v_{\alpha}\left(a_{2}\right)\right\}$, for any $t_{1}, t_{2} \in Y$, we have $\Theta_{\text {sup }}\left(\mu_{\alpha}\right)\left(t_{1}\right)=\sup \left\{\mu_{\alpha}(a)\right.$ | $\left.a \in \Theta^{-1}\left(t_{1}\right)\right\} \geq \sup \left\{\min \left\{\mu_{\alpha}\left(a_{1} \bullet a_{2}\right), \mu_{\alpha}\left(a_{2}\right)\right\} \mid a_{1} \cdot a_{2} \in\right.$ $\Theta^{-1}\left(t_{1} \cdot t_{2}\right)$ and $\left.a_{2} \in \Theta^{-1}\left(t_{2}\right)\right\}=\min \left\{\sup \left\{\mu_{\alpha}\left(a_{1} \cdot a_{2}\right)\right.\right.$ | $\left.\left.a_{1} \cdot a_{2} \in \Theta^{-1}\left(t_{1} \cdot t_{2}\right)\right\}, \sup \left\{\mu_{\alpha}\left(a_{2}\right) \quad \mid \quad a_{2} \in \Theta^{-1}\left(t_{2}\right)\right\}\right\}$ $=\min \left\{\Theta_{\text {sup }}\left(\mu_{\alpha}\right)\left(t_{1} \cdot t_{2}\right), \Theta_{\text {sup }}\left(\mu_{\alpha}\right)\left(t_{2}\right)\right\}$. Also, $\Theta_{\text {sup }}\left(\nu_{\alpha}\right)\left(t_{1}\right)=$ $\sup \left\{v_{\alpha}(a) \mid a \in \Theta^{-1}\left(t_{1}\right)\right\} \leq \sup \left\{\max \left\{v_{\alpha}\left(a_{1} \cdot a_{2}\right), v_{\alpha}\left(a_{2}\right)\right\} \mid\right.$ $a_{1} \bullet a_{2} \in \Theta^{-1}\left(t_{1} \bullet t_{2}\right)$ and $\left.a_{2} \in \Theta^{-1}\left(t_{2}\right)\right\}=\max \left\{\sup \left\{v_{\alpha}\left(a_{1} \bullet a_{2}\right) \mid\right.\right.$ $\left.\left.a_{1} \cdot a_{2} \in \Theta^{-1}\left(t_{1} \cdot t_{2}\right)\right\}, \sup \left\{v_{\alpha}\left(a_{2}\right) \mid a_{2} \in \Theta^{-1}\left(t_{2}\right)\right\}\right\}=$ $\max \left\{\Theta_{\text {sup }}\left(\nu_{\alpha}\right)\left(t_{1} \bullet t_{2}\right), \Theta_{\text {sup }}\left(\nu_{\alpha}\right)\left(t_{2}\right)\right\}$. Thus we consider that $\Theta(\alpha)=\prec b,\left(\Theta_{\text {sup }}\left(\mu_{\alpha}\right), \Theta_{\text {inf }}\left(\nu_{\alpha}\right)\right)>$ is $(I F \rho i)$ of $Y$.

Theorem 28. Assume $\Theta: \mathfrak{R} \longrightarrow Y$ is $\rho$-homomorphism of $\rho$-algebra $\mathfrak{R}$ into $\rho$-algebra $Y$ and $\alpha=\left\{<a,\left(\mu_{\alpha}(a), v_{\alpha}(a)\right)>\mid\right.$ $a \in \mathfrak{R}\}$ is (IF $\bar{\rho} i)$ of $\mathfrak{R}$. Then $\Theta(\alpha)=\prec b,\left(\Theta_{\text {sup }}\left(\mu_{\alpha}\right), \Theta_{\text {inf }}\left(\nu_{\alpha}\right)\right)>$ is $(I F \bar{\rho} i)$ of $Y$.

Proof. Since $\alpha=\left\{\left\langle a,\left(\mu_{\alpha}(a), \nu_{\alpha}(a)\right)\right\rangle \mid a \in \mathfrak{R}\right\}$ is (IF $\bar{\rho} i$ ) of $\mathfrak{R}$. Then by Theorem 26 and Remark 14 we have $\Theta(\alpha)=\prec b,\left(\Theta_{\text {sup }}\left(\mu_{\alpha}\right), \Theta_{\text {inf }}\left(\nu_{\alpha}\right)\right)>$ as (IF $\left.\rho s\right)$ of $Y$. Hence condition (2) in Definition 12 is held. Assume that $0_{\mathfrak{R}}$ and $0_{Y}$ are constants of $\rho$ - algebras $\Re$ and $Y$, respectively. Since $\alpha=\left\{\left\langle a,\left(\mu_{\alpha}(a), \nu_{\alpha}(a)\right)>\right| a \in \mathfrak{R}\right\}$ is $(I F \bar{\rho} i)$ of $\mathfrak{R}$, hence $\mu_{\alpha}\left(0_{\mathfrak{R}}\right) \geq \mu_{\alpha}(a)$ and $\nu_{\alpha}\left(0_{\mathfrak{R}}\right) \leq \nu_{\alpha}(a), \forall a \in \mathfrak{R}$. Since $\Theta$ is $\rho$-homomorphism of $\rho$-algebras, then $\Theta\left(0_{\mathfrak{R}}\right)=0_{Y}$, where $0_{\Re}$ and $0_{Y}$ are constants for $\rho$-algebras $\Re$ and $Y$, respectively. Noticing that $0_{\Re} \in \Theta^{-1}\left(0_{Y}\right)$ and $\left\{a \mid a \in \Theta^{-1}(b)\right\} \subseteq$ $\{a \mid a \in \mathfrak{R}\}$ for any $b \in Y$, then we have $\Theta_{\text {sup }}\left(\mu_{\alpha}\right)\left(0_{Y}\right)=$ $\sup \left\{\mu_{\alpha}(a) \mid a \in \Theta^{-1}\left(0_{Y}\right)\right\}=\mu_{\alpha}\left(0_{\Re}\right) \geq \sup \left\{\mu_{\alpha}(a) \mid a \in\right.$ $\Re\} \geq \sup \left\{\mu_{\alpha}(a) \mid a \in \Theta^{-1}(b)\right\}=\Theta_{\text {sup }}\left(\mu_{\alpha}\right)(b)$. Also, $\Theta_{\text {sup }}\left(\nu_{\alpha}\right)\left(0_{Y}\right)=\inf \left\{\nu_{\alpha}(a) \mid a \in \Theta^{-1}\left(0_{Y}\right)\right\}=v_{\alpha}\left(0_{\Re}\right) \leq$ $\inf \left\{\nu_{\alpha}(a) \mid a \in \Re\right\} \leq \inf \left\{\nu_{\alpha}(a) \mid a \in \Theta^{-1}(b)\right\}=\Theta_{\text {sup }}\left(\nu_{\alpha}\right)(b)$. Hence $\Theta(\alpha)=<b,\left(\Theta_{\text {sup }}\left(\mu_{\alpha}\right), \Theta_{\text {inf }}\left(\nu_{\alpha}\right)\right)>$ is $(I F \bar{\rho} i)$ of $Y$.

\section{Some Applications on $I F \rho_{S}(\mathfrak{R})$}

In this section, some applications on $\operatorname{IF} \rho_{S}(\mathfrak{R})$ are shown like the binary relations $\underset{\mu}{\approx}, \underset{v}{\approx}$ and $\Gamma_{r}$ on $\operatorname{IF} \rho_{S}(\mathfrak{R})$. Also, in this section the equivalence classes for theses binary relations are given, and some of their basic properties are studied.

\subsection{Equivalence Classes Modulo $(\underset{\mu}{\approx} / \approx)$. Denote the collection} of all $\left(I F \rho_{S}\right)$ of $\mathfrak{R}$ by $I F \rho_{S}(\mathfrak{R})$ and let $r \in[0,1]$. Define binary relations $\underset{\mu}{\approx}$ and $\underset{v}{\approx}$ on $I F \rho_{S}(\Re)$ as follows.

$\alpha \underset{\tilde{\mu}}{\approx} \beta \Longleftrightarrow W\left(\mu_{\alpha}, r\right)=W\left(\mu_{\beta}, r\right)$ and $\alpha \underset{v}{\approx} \beta \Longleftrightarrow$ $L\left(\nu_{\alpha}, r\right)=L\left(\nu_{\beta}, r\right)$, respectively, for $\alpha=\left\langle a, \mu_{\alpha}, \nu_{\alpha}>\right.$ and $\beta=\prec a, \mu_{\beta}, v_{\beta}>$ in $\operatorname{IF} \rho_{S}(\mathfrak{R})$. Moreover, it is clear that $\underset{\mu}{\approx}$ and $\approx \widetilde{v}$ are equivalence relations on $\operatorname{IF} \rho_{S}(\Re)$. If $\alpha=\prec a, \mu_{\alpha}, \nu_{\alpha}>\epsilon$ $I F \rho_{S}(\mathfrak{R})$, then we refer to the equivalence class of $\alpha=\prec$ $a, \mu_{\alpha}, v_{\alpha}>$ modulo $\underset{\mu}{\approx}$ (resp., $\underset{v}{\approx}$ ) by $\langle\alpha\rangle_{\mu}$ (resp., $\langle\alpha\rangle_{\nu}$ ), and we refer to the family of all equivalence classes of $\alpha$ modulo $\approx$

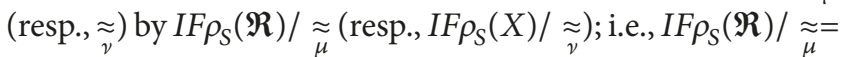
$\left\{\langle\alpha\rangle_{\mu} \mid \alpha=<a, \mu_{\alpha}, \nu_{\alpha}>\in I F \rho_{S}(\mathfrak{R})\right\}$ (resp., $I F \rho_{S}(\mathfrak{R}) / \approx=$ $\left.\left\{\langle\alpha\rangle_{\mu}\left|\alpha=\prec a, \mu_{\alpha}, v_{\alpha}\right\rangle \in I F \rho_{S}(\Re)\right\}\right)$. Moreover, denote the collection of all $\rho$-ideals of $\mathfrak{R}$ by $\rho_{I}(\mathfrak{R})$ and let $r \in[0,1]$. Let $\sigma_{r}$ and $\eta_{r}$ be maps from $I F \rho_{S}(\Re)$ to $\rho_{I}(\Re) \cup\{\phi\}$ by $\sigma_{r}(\alpha)=W\left(\mu_{\alpha}, r\right)$ and $\eta_{r}(\alpha)=L\left(\nu_{\alpha}, r\right)$, respectively, $\forall \alpha=\prec$ $a, \mu_{\alpha}, v_{\alpha}>\in I F \rho_{S}(\Re)$. In other words, $\sigma_{r}$ and $\eta_{r}$ are welldefined.

Theorem 29. Let $\sigma_{r}$ and $\eta_{r}$ be the maps from $I F \rho_{S}(\Re)$ to $\rho_{I}(\Re) \cup\{\phi\}$. Then $\sigma_{r}$ and $\eta_{r}$ are surjective, for each $r \in(0,1)$. 
Proof. Let $r \in(0,1)$. Then $\overline{0}=\left\langle a, \overline{0}, \overline{1}>\right.$ is in $\operatorname{IF} \rho_{S}(\mathfrak{R})$, where each one of $\overline{0}$ and $\overline{1}$ is (FS) in $\Re$ defined by $\overline{0}(a)=0$ and $\overline{1}(a)=1, \forall a \in \Re$. Furthermore, $\sigma_{r}(\widehat{0})=W(\overline{0}, r)=\phi=$ $L(\overline{1}, r)=\eta_{r}(\overline{0})$. Let $\phi \neq H \in \rho_{I}(\Re) . \forall a \in \Re$, let $\mu_{H}(a)=$ $\left\{\begin{array}{l}1, \text { if } a \in H \\ 0, \text { if } a \notin H\end{array}\right.$, and $\nu_{H}(a)=1-\mu_{H}(a)$; thus $\sigma_{r}(\widehat{H})=W\left(\mu_{H}, r\right)=$ $H=L\left(v_{H}, r\right)=\eta_{r}(\widehat{H})$. Now, we want to prove that $\widehat{H}=<$ $x, \mu_{H}, \nu_{H}>\in \operatorname{IF} \rho_{S}(\Re)$. Since $H \in \rho_{I}(\Re)$, then by condition (1) in Definition 5 we have $H$ as $\rho$-subalgebra of $\Re$ and this implies that $W\left(\mu_{H}, r\right)$ and $L\left(\nu_{H}, r\right)$ are $\rho$-subalgebras of $\Re$. By Theorem 22 we consider $\bar{H}=<a, \mu_{H}, \nu_{H}>\in \operatorname{IF} \rho_{S}(\Re)$. Therefore, $\forall H \in \rho_{I}(\mathfrak{R})$ we consider $\sigma_{r}(\widetilde{H})=H$ and $\eta_{r}(\widetilde{H})=$ $H$ for some $\widehat{H} \in I F \rho_{S}(\Re)$. This completes the proof.

Theorem 30. Let $I F \rho_{S}(\mathfrak{R}) / \approx \widetilde{\tilde{\mu}}$ and $I F \rho_{S}(\mathfrak{R}) / \approx$ be quotient sets. Then they are equipotent to $\rho_{I}(\mathfrak{R}) \cup\{\phi\}, \forall r \in(0,1)$.

Proof. Assume $r \in(0,1)$ and let $\sigma_{r}^{\prime}\left(\right.$ resp. $\left.\eta_{r}^{\prime}\right)$ be a map from $I F \rho_{S}(\mathfrak{R}) / \approx \widetilde{\tilde{\mu}}\left(\right.$ resp., $I F \rho_{S}(\mathfrak{R}) / \underset{\tilde{\nu}}{\text { ) }}$ to $\rho_{I}(\mathfrak{R}) \cup\{\phi\}$ and they are defined by $\sigma_{r}^{\prime}\left(\langle\alpha\rangle_{\mu}\right)=\sigma_{r}(\alpha)\left(\right.$ resp. $\left.\eta_{r}^{\prime}\left(\langle\alpha\rangle_{\nu}\right)=\eta_{r}(\alpha)\right)$, $\forall \alpha=<a, \mu_{\alpha}, v_{\alpha}>\in \operatorname{IF} \rho_{S}(\Re)$. Hence, $\alpha \underset{\tilde{\mu}}{\approx} \beta$ and $\alpha \approx \widetilde{\tilde{v}} \beta$, $\forall \alpha=\left\langle a, \mu_{\alpha}, v_{\alpha}>\right.$ and $\beta=\left\langle a, \mu_{\beta}, v_{\beta}>\right.$ in $\operatorname{IF} \rho_{S}(\mathfrak{R})$, if $W\left(\mu_{A}, r\right)=W\left(\mu_{B}, r\right)$ and $L\left(\nu_{A}, r\right)=L\left(\nu_{B}, r\right)$. Then $\langle\alpha\rangle_{\mu}=$ $\langle\beta\rangle_{\mu}$ and $\langle\alpha\rangle_{\nu}=\langle\beta\rangle_{v}$. This implies the maps $\sigma_{r}^{\prime}$ and $\eta_{r}^{\prime}$ are injective. Moreover, let $\phi \neq H \in \rho_{I}(\mathfrak{R})$ and $\forall a \in \Re$, let

$$
\mu_{H}(a)= \begin{cases}1, & \text { if } a \in H \\ 0, & \text { if } a \notin H,\end{cases}
$$

$v_{H}(a)=1-\mu_{H}(a)$, and thus $\widehat{H}=<a, \mu_{H}, v_{H}>\in \operatorname{IF} \rho_{S}(\Re)$. We consider that $\sigma_{r}^{\prime}\left(\langle\widehat{H}\rangle_{\mu}\right)=\sigma_{r}(\widehat{H})=W\left(\mu_{H}, r\right)=H$, and $\eta_{r}^{\prime}\left(\langle\widehat{H}\rangle_{\nu}\right)=\eta_{r}(\widehat{H})=L\left(\nu_{H}, r\right)=H$. Finally, for $\widehat{0}=<$ $a, \overline{0}, \overline{1}>\in \operatorname{IF} \rho_{S}(\Re)$ we have $\sigma_{r}^{\prime}\left(\left\langle\langle\hat{0}\rangle_{\mu}\right)=\sigma_{r}(\overline{0})=W(\overline{0}, r)=\phi\right.$ and $\eta_{r}^{\prime}\left(\langle\widehat{0}\rangle_{\nu}\right)=\eta_{r}(\widehat{0})=L(\overline{1}, r)=\phi$. Therefore $\sigma_{r}^{\prime}$ and $\eta_{r}^{\prime}$ are surjective, and we are done.

4.2. Equivalence Class Modulo $\Gamma_{r}$. Another relation $\Gamma_{r}$ on $\operatorname{IF} \rho_{S}(\mathfrak{R})$ is defined by $(\alpha, \beta) \in \Gamma_{r} \Longleftrightarrow W\left(\mu_{\alpha}, r\right) \cap L\left(\nu_{\alpha}, r\right)=$ $W\left(\mu_{\beta}, r\right) \cap L\left(v_{\beta}, r\right), \forall r \in[0,1]$ and, $\forall \alpha=\left\langle a, \mu_{\alpha}, v_{\alpha}\right\rangle$, $\beta=\left\langle a, \mu_{\beta}, v_{\beta}>\in \operatorname{IF} \rho_{S}(\mathfrak{R})\right.$. Moreover, the relation $\Gamma_{r}$ is also an equivalence relation on $I F \rho_{S}(\Re)$. Let $\langle\alpha\rangle_{\Gamma_{r}}$ denote the equivalence class of $\alpha=\left\langle a, \mu_{\alpha}, v_{\alpha}\right\rangle$ modulo $\Gamma_{r}, \forall \alpha=<$ $a, \mu_{\alpha}, v_{\alpha}>\in I F \rho_{S}(\Re)$.

Theorem 31. For any $r \in(0,1)$, the map $\psi_{r}: \operatorname{IF} \rho_{S}(\Re) \longrightarrow$ $\rho_{I}(\mathfrak{R}) \cup\{\phi\}$ defined by $\psi_{r}(\mathfrak{R})=\sigma_{r}(\mathfrak{R}) \cap \eta_{r}(\mathfrak{R}), \forall \alpha=<$ $a, \mu_{\alpha}, \nu_{\alpha}>\in \operatorname{IF} \rho_{S}(\Re)$ is surjective.

Proof. Let $r \in(0,1)$. For $\overline{0}=<a, \overline{0}, \overline{1}>\in \operatorname{IF} \rho_{S}(\Re)$, we get $\psi_{r}(\widehat{0})=\sigma_{r}(\overline{0}) \cap \eta_{r}(\overline{0})=W(\overline{0}, r) \cap L(\overline{1}, r)=\phi$. For any $H \epsilon$ $\operatorname{IF} \rho_{S}(\mathfrak{R})$, there exists $\widehat{H}=\prec a, \mu_{H}, v_{H}>\in \operatorname{IF} \rho_{S}(\mathfrak{R})$, where

$$
\mu_{H}(a)= \begin{cases}1, & \text { if } a \in H \\ 0, & \text { if } a \notin H\end{cases}
$$

and $\nu_{H}(a)=1-\mu_{H}(a)$ such that $\psi_{r}(\widehat{H})=\sigma_{r}(\widehat{H}) \cap \eta_{r}(\widehat{H})=$ $W\left(\mu_{H}, r\right) \cap L\left(\nu_{H}, r\right)=H$. This completes the proof.
Theorem 32. For any $r \in(0,1)$, the quotient $\operatorname{set} \operatorname{IF} \rho_{S}(\mathfrak{R}) / \Gamma_{r}$ is equipotent to $\rho_{I}(X) \cup\{\phi\}$.

Proof. Assume $r \in(0,1)$ and $\psi_{r}^{\prime}: I F \rho_{S}(\Re) / \Gamma_{r} \longrightarrow \rho_{I}(\mathfrak{R}) \cup\{\phi\}$ is a map defined by $\psi_{r}^{\prime}\left(\langle\alpha\rangle_{\Gamma_{r}}\right)=\psi_{r}(\alpha), \forall\langle\alpha\rangle_{\Gamma_{r}} \in \operatorname{IF} \rho_{S}(\Re) / \Gamma_{r}$.

Suppose that $\psi_{r}^{\prime}\left(\langle\alpha\rangle_{\Gamma_{r}}\right)=\psi_{r}^{\prime}\left(\langle\beta\rangle_{\Gamma_{r}}\right)$ for any $\langle\alpha\rangle_{\Gamma_{r}},\langle\beta\rangle_{\Gamma_{r}} \in$ $\operatorname{IF} \rho_{S}(\Re) / \Gamma_{r}$. We consider that $\sigma_{r}(\alpha) \cap \eta_{r}(\alpha)=\sigma_{r}(\beta) \cap$ $\eta_{r}(\beta)$, i.e., $W\left(\mu_{\alpha}, r\right) \cap L\left(\nu_{\alpha}, r\right)=W\left(\mu_{\beta}, r\right) \cap L\left(\nu_{\beta}, r\right)$. Hence $(\alpha, \beta) \in \Gamma_{r}$, and so $\langle\alpha\rangle_{\Gamma_{r}}=\langle\beta\rangle_{\Gamma_{r}}$. Therefore $\psi_{r}^{\prime}$ is injective. Furthermore, for $\overline{0}=\langle a, \overline{0}, \overline{1}\rangle \in \operatorname{IF} \rho_{S}(\Re)$ we get $\psi_{r}^{\prime}\left(\langle\overline{0}\rangle_{\Gamma_{r}}\right)$ $=\psi_{r}(\widehat{0})=\sigma_{r}(\overline{0}) \cap \eta_{r}(\widehat{0})=W(\overline{0}, r) \cap L(\overline{1}, r)=\phi$. Let $\widehat{H}=<$ $a, \mu_{H}, \nu_{H}>\in I F \rho_{S}(\Re), \forall H \in I F \rho_{S}(\Re)$, be the same $\left(I F \rho_{S}\right)$ of $X$ that is defined in the proof of Theorem 22. Then we have $\psi_{r}^{\prime}\left(\langle\widehat{H}\rangle_{\Gamma_{r}}\right)=\psi_{r}(\widehat{H})=\sigma_{r}(\widehat{H}) \cap \eta_{r}(\widehat{H})=W\left(\mu_{H}, r\right) \cap L\left(\nu_{H}, r\right)=$ $H$. Hence $\psi_{r}^{\prime}$ is surjective. This completes the proof.

\section{Conclusion}

In this work, we introduce the notions of (IF $\rho)$, (IF $\rho$ i), $(I F \bar{\rho} i)$, and others; then we proved that for any $\rho$-subalgebra of $X$ can be considered as both $\mu$-level $\rho$-subalgebra and $\nu$-level $\rho$-subalgebra of some (IF $\rho s)$ of $\mathfrak{R}$. At the same time, we proved that intersection of any family of (IF $\rho s)$ of $X$ is $(I F \bar{\rho} i)$ of $X$. Also, we show that if IFS $\alpha=\{<$ $\left.a,\left(\mu_{\alpha}(a), v_{\alpha}(a)\right)>\mid a \in \Re\right\}$ of $\rho$ - algebra $X$ such that the sets $W\left(\mu_{\alpha}, r\right)$ and $L\left(\nu_{\alpha}, r\right)$ are $\rho$-subalgebras of $\mathfrak{R}$. Then $\alpha$ is (IF $\rho$ ) of $\mathfrak{R}$. Further, some interesting theorems about $\rho$-homomorphism are given. Finally, some binary relations $\underset{\tilde{\mu}}{\widetilde{v}} \approx \vec{v}$ and $\Gamma_{r}$ on $\operatorname{IF}_{S}(\mathfrak{R})$ are obtained, and some of their basic properties are discussed. In future work, we will investigate IF in new types of algebras like $B C L^{+}$-algebras, $B C L^{+}$-subalgebras, $B C L^{+}$-ideals and others. Next, we will study their characteristics.

\section{Data Availability}

Data used to support the findings of this study are available from the corresponding author upon request.

\section{Conflicts of Interest}

The authors declare no conflicts of interest.

\section{References}

[1] L. A. Zadeh, "Fuzzy sets," Information and Control, vol. 8, no. 3, pp. 338-353, 1965.

[2] M. Shabir and M. I. Ali, "Soft ideals and generalized fuzzy ideals in semigroups," New Mathematics and Natural Computation, vol. 5, no. 3, pp. 599-615, 2009.

[3] A. Aygünoğlu and H. Aygün, "Introduction to fuzzy soft groups," Computers \& Mathematics with Applications, vol. 58, no. 6, pp. 1279-1286, 2009.

[4] S. Mahmood, "Dissimilarity fuzzy soft points and their applications," Fuzzy Information and Engineering, vol. 8, no. 3, pp. 281-294, 2016. 
[5] P. K. Maji, R. Biswas, and A. R. Roy, "Fuzzy soft sets," Journal of Fuzzy Mathematics, vol. 9, no. 3, pp. 589-602, 2001.

[6] K. T. Atanassov, "Intuitionistic fuzzy sets," Fuzzy Sets and Systems, vol. 20, no. 1, pp. 87-96, 1986.

[7] P. A. Ejegwa, A. J. Akubo, and O. M. Joshua, "Intuitionistic fuzzy sets and its application in career determination via normalized euclidean distance method," European Scientific Journal, vol. 10, no. 12, pp. 349-365, 2014.

[8] S. M. Khalil, "On intuitionistic fuzzy soft $b$-closed sets in intuitionistic fuzzy soft topological spaces," Annals of Fuzzy Mathematics and Informatics, vol. 10, no. 2, pp. 221-233, 2015.

[9] P. K. Maji, R. Biswas, and A. R. Roy, "Intuitionistic fuzzy soft sets," Journal of Fuzzy Mathematics, vol. 9, no. 3, pp. 677-692, 2001.

[10] S. Mahmood and Z. Al-Batat, "Intuitionistic fuzzy soft LAsemigroups and intuitionistic fuzzy soft ideals," International Journal of Applications of Fuzzy Sets and Artificial Intelligence, vol. 6, pp. 119-132.

[11] Y. B. Jun, H. S. Kim, and D. S. Yoo, "Intuitionistic fuzzy $d$ algebras," Scientiae Mathematicae Japonicae, vol. 66, no. 1, pp. $117-125,2007$.

[12] Y. Imai and K. Iseki, "On axiom systems of propositional calculi, XIV," Proceedings of the Japan Academy, Series A: Mathematical Sciences, vol. 42, no. 1, pp. 19-22, 1966.

[13] J. Neggers and H. S. Kim, "On d-algebras," Mathematica Slovaca, vol. 49, no. 1, pp. 19-26, 1999.

[14] S. Mahmood and M. A. Alradha, "Characterizations of $\rho$ algebra and generation permutation topological $\rho$-algebra using permutation in symmetric group," American Journal of Mathematics and Statistics, vol. 7, no. 4, pp. 152-159, 2017.

[15] S. Mahmood and M. A. Alradha, "Soft Edge $\rho$-Algebras of the power sets," International Journal of Applications of Fuzzy Sets and Artificial Intelligence, vol. 7, pp. 231-243, 2017.

[16] H. Aktas and N. Cagman, "Soft sets and soft groups," Information Sciences, vol. 177, no. 13, pp. 2726-2735, 2007. 


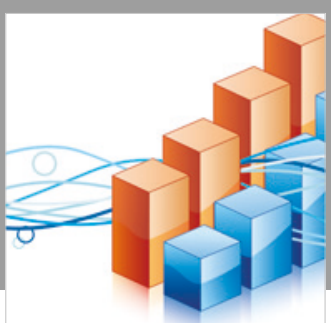

Advances in

Operations Research

\section{-n-m}
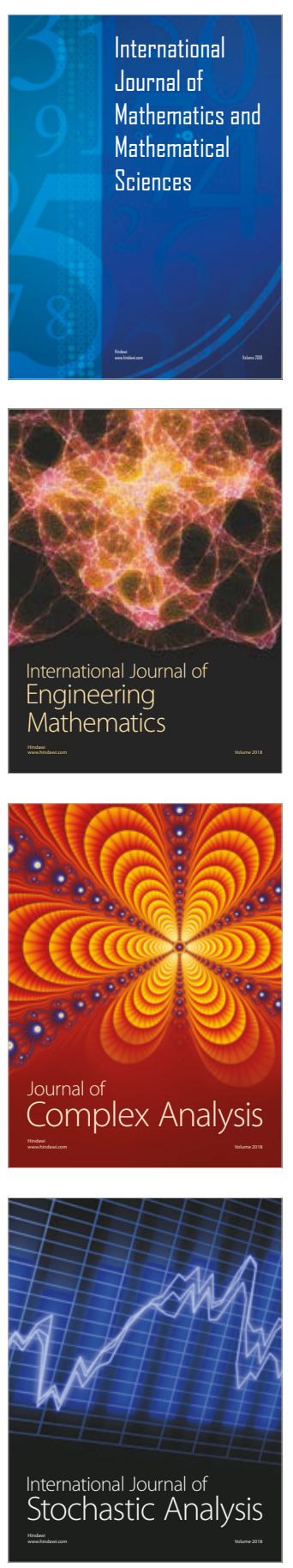
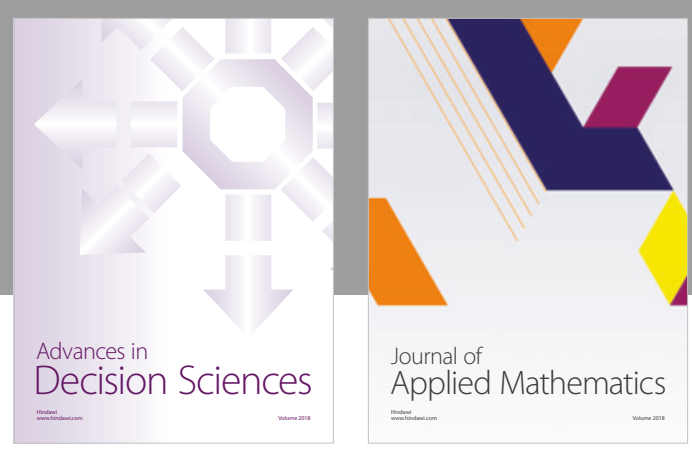

Journal of

Applied Mathematics
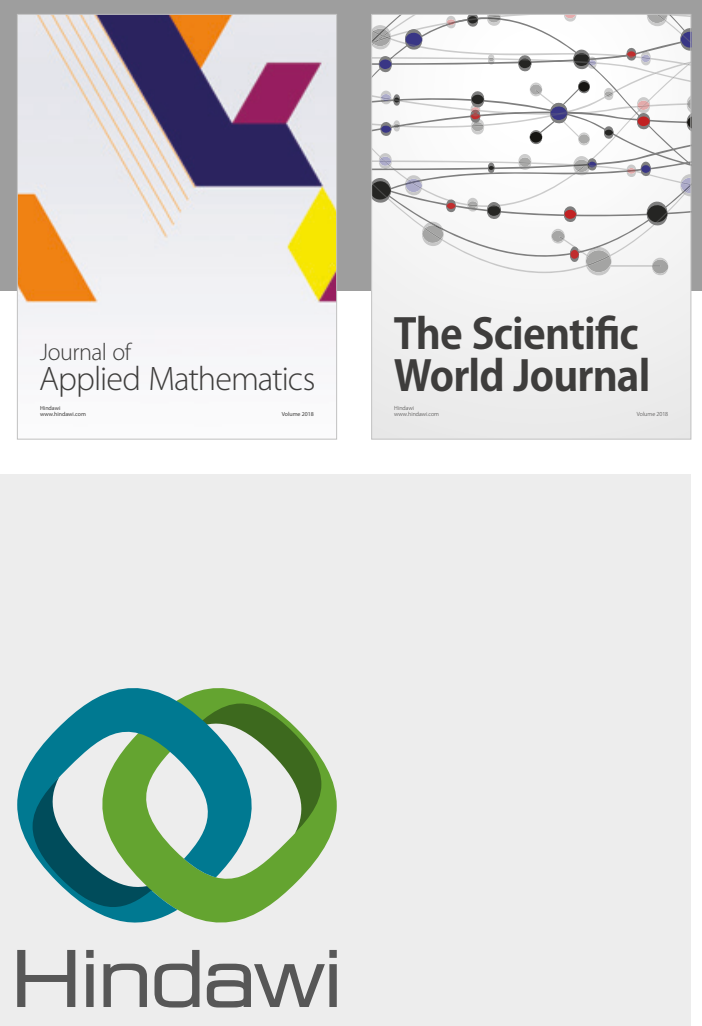

Submit your manuscripts at

www.hindawi.com

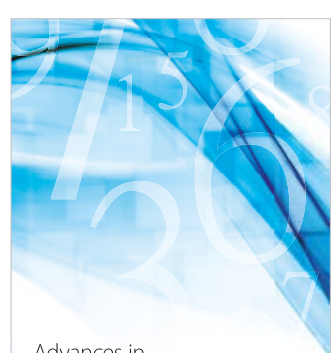

Advances in
Numerical Analysis
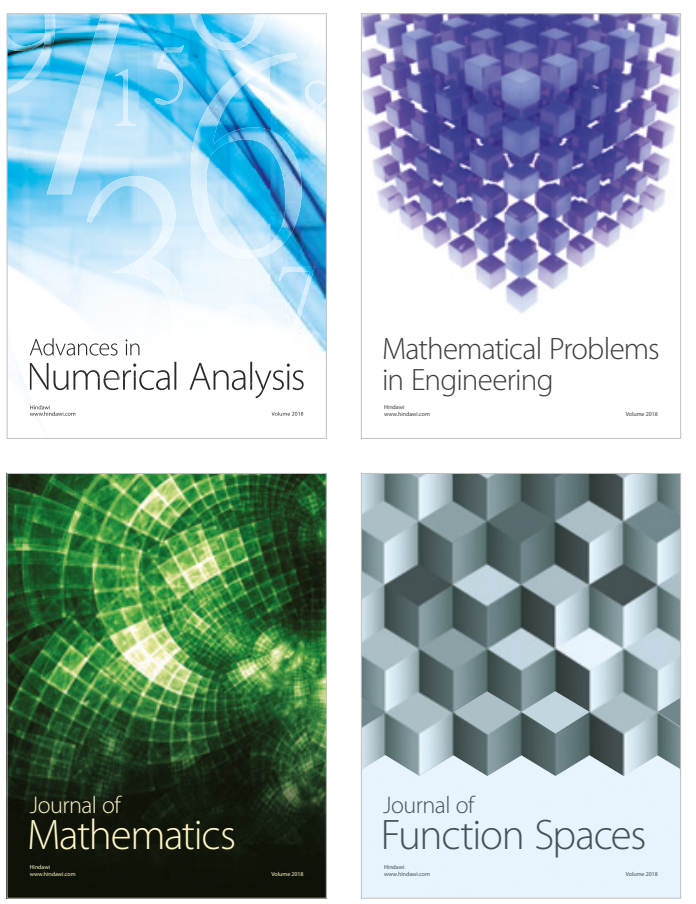

Mathematical Problems in Engineering

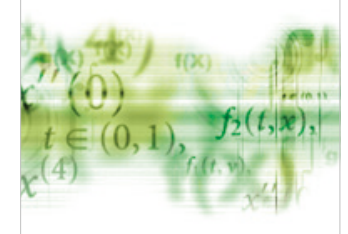

International Journal of

Differential Equations

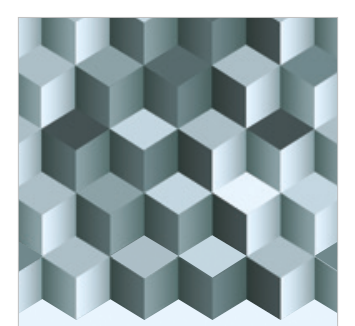

Journal of

Function Spaces

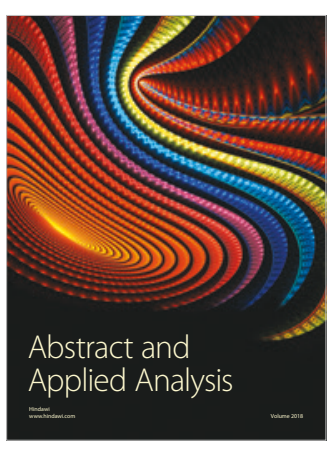

The Scientific

World Journal

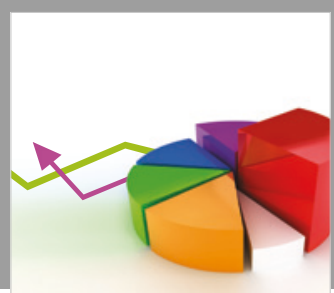

Journal of

Probability and Statistics
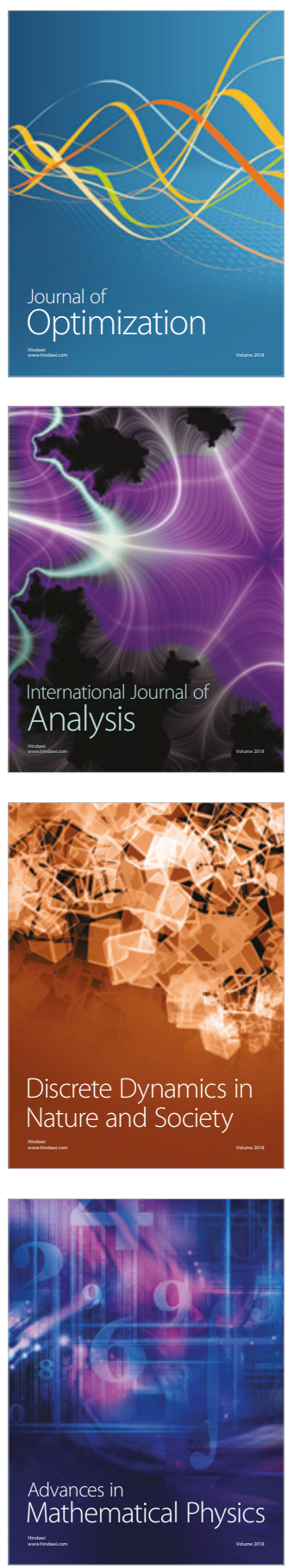